\title{
CONDICIONES QUE FAVORECEN EL EJERCICIO PROFESIONAL: EXPLORACIÓN DE LAS EXPERIENGIAS DE FISIOTERAPEUTAS EGRESADOS DE LA UNIVERSIDAD CES, MEDELLÍN ${ }^{1}$
}

\author{
CONDITIONS THAT FAVOR THE PROFESSIONAL EXERCISE: EXPLORATION OF \\ PHYSIOTHERAPISTS'S EXPERIENGES DEGREE OF THE UNIVERSIDAD CES, MEDELLÍN
}

\author{
Verónica Mariela Tamayo ${ }^{2}$ \\ Catalina Betancur Betancur ${ }^{3}$ \\ Luisa Alejandra Vélez Castañeda ${ }^{4}$
}

Fecha de Recepción: 06 de junio de 2016

Fecha de Aceptación: 18 de diciembre de 2016

Citar como:

Tamayo V, Betancur-Betancur C, Vélez-Castañeda L. (2016). Condiciones que favorecen el ejercicio profesional: exploración de las experiencias de fisioterapeutas egresados de la Universidad CES, Medellín. Revista Movimiento Científico 10(2): 30-40.

Tamayo V, Betancur-Betancur C, Vélez-Castañeda L. (2016). Condiciones que favorecen el ejercicio profesional: exploración de las experiencias de fisioterapeutas egresados de la Universidad CES, Medellín. Revista Movimiento Científico [en línea] 2016, [fecha de consulta: dd//mm/aaaa]; 10(2): 30-40. Disponible desde: http://revistas.iberoamericana.edu.co/index.php/Rmcientifico/issue/archive

\section{RESUMEN}

Introducción: la historia de la fisioterapia le ha permitido posicionarse como una profesión y un estilo de vida. En Colombia la fisioterapia ha cobrado sentido desde sus inicios con un papel importante en la rehabilitación de la población y sobre todo por la satisfacción que sienten los profesionales al realizarla; en Antioquia existen solo 2 universidades encargadas de la formación de fisioterapeutas, de estas dos hace parte la universidad CES que ha venido formando profesionales humanos y sobre todo excelentes desde su primera promoción hasta la actualidad. La cantidad de profesionales egresados de la universidad CES ha aumentado gradualmente y por eso se hace necesario conocer las condiciones que favorecen su ejercicio profesional, a fin de mantener una apuesta formativa pertinente y adecuada al contexto. Objetivo: explorar las condiciones de trabajo en el ejercicio profesional de los fisioterapeutas egresados de la Universidad CES. Materiales y métodos: estudio cualitativo de alcance exploratorio. Se realizó una entrevista semiestructurada y un grupo focal con cuatro egresados; el análisis de la información orientó por las indicaciones metodológicas que la teoría fundada ofrece en términos de codificación de la información. Resultados: para los

1 Artículo original de investigación derivado del Estudio Nacional sobre las condiciones de trabajo y salud de los fisioterapeutas en Colombia; los autores declaran que no existen conflictos de interés.

2 Magíster en Educación y Desarrollo Humano. Magíster en Dirección. Investigadora. Facultad de Fisioterapia. Universidad CES. Correo electrónico: vtamayo@ces.edu.co.

Magíster en investigación psicoanalítica. Docente investigadora. Facultad de Psicología. Universidad CES.

Estudiante de Fisioterapia, Universidad CES. 
participantes, hay cuatro condiciones que favorecen el ejercicio profesional: cualificación profesional permanente; construcción del rol profesional; condiciones de trabajo y amor por la profesión. Estas condiciones se ven mejoradas gracias al empoderamiento, lo que pone en el horizonte la cuestión del gremio y lo que la formación debe ofrecer para el logro de la participación y la vinculación activa en la construcción del rol profesional. Conclusiones: las condiciones que favorecen el ejercicio profesional de los fisioterapeutas en la universidad CES están asociadas no solamente a lo externo, sino también a lo personal como lo es sentir amor por lo que se hace y donde se hace, estar y sentirse satisfecho con la vida laboral.

Palabras clave: Fisioterapia, Graduados, Práctica profesional, Movilidad Laboral.

\section{ABSTRACT}

Background: Physical therapy history has allowed itself to position as a profession and a life-style. In Colombia, physical therapy since its early years has played an important role in the rehabilitation of the population, especially for how physical therapists are satisfied with their profession. In Antioquia there are only two universities in charge of the training of physical therapists, of which Universidad CES in one. To date, and since its foundation, Universidad CES is committed to training of humane and excellent professionals. The quantity of alumni from Universidad CES have been gradually increasing; thus, it is necessary to know the conditions that favour the professional exercise, with the goal of maintaining a physical therapy program that is pertinent and appropriate to the context. Objective: investigate the job conditions of the physical therapists' alumni from Universidad CES. Materials and methods: A qualitative exploratory. Four semi-structured interviews and a focus group were conducted with Universidad CES alumni. The information analysis was based on grounded theory for the coding of the information. Results: For the participants there are four conditions that foster their professional exercise: continuous professional qualification, building the professional role, job conditions, and love for their profession. There is an improvement in these conditions thanks to the empowerment, which puts in the horizon the issue of the professional field and what training has to offer to the achieve the participation and active involvement in the construction of the professional role. Conclusions: The conditions that favour the professional exercise of physical therapists at Universidad CES are associated not only to the external conditions but to the personal factors such as love to what they do and where they do it, being and feeling satisfied with their work life.

Keywords: Physical Therapy. Graduates. Professional Practice. Career Mobility.

\section{INTRODUCGIÓN}

"El término Fisioterapia, atendiendo a su significado y sentido etimológico, permite identificar una de las formas más antiguas con las que el ser humano ha pretendido combatir la enfermedad en las diversas culturas" (Chillón, Rebollo, \& Meroño, 2008); de ello se encuentran precedentes lejanos en el tiempo que muestran cómo la fisioterapia es una de las profesiones del área de la salud con mayor impacto en la población en cualquier momento del ciclo vital (Cerpa, 2010).

En las dos décadas anteriores en Colombia se ha evidenciado un aumento de la oferta educativa, pasando de tener 5 instituciones que ofertaban este pregrado en la década de los años ochenta, a 31 programas activos ofertados en el año 2016 según datos del SNIES (Ministerio de Educación Nacional, 2016). Esto significa que ha habido un gran aumento de programas de formación, al igual que el número de fisioterapeutas por habitante: pasando de 0,07 fisioterapeutas/1000 habitantes en el año 1984 a 0,48 fisioterapeutas/1000 habitantes en el año 2000; para 2016 la relación es de 0.8 fisioterapeutas/1000 habitantes (Servicio Nacional de Aprendizaje, SENA, 2008). Es necesario mencionar que internacionalmente debe existir un fisioterapeuta por cada 1000 habitantes y según lo anterior, en Colombia son aún insuficientes 
los fisioterapeutas para la atención a la población del país.

La fisioterapia surge como respuesta a las necesidades en salud, sociales y de desarrollo de las poblaciones; a ello se suman las enfermedades que generan discapacidad y disminuyen la calidad de vida y del aumento de la producción e industrialización de las poblaciones; esta profesión se ocupa de la intervención en el movimiento corporal humano como su objeto de estudio dentro del equipo de rehabilitación integral (Gallego, 2007).

La configuración de la fisioterapia como profesión surge en el siglo XX ligada a momentos históricos como la Segunda Guerra Mundial y la epidemia de poliomielitis de mitad de ese siglo, para comenzar una nueva historia basada en las características propias de una profesión, como son la vida universitaria, las leyes que la sustentan y el reconocimiento dentro del sistema de salud.

En la década de los años 90 del siglo XX a la par con la nueva Constitución Política de Colombia se dio la transformación del modelo de salud colombiano y la reforma de la educación superior, generándose cambios en los modelos de educación y salud que acercaron las comunidades profesional y académica, para propiciar el desarrollo profesional y actualizar la pertinencia social del rol del fisioterapeuta en el país.

En 1999, luego de años de trabajo gremial, se aprobó la Ley 528 en que la fisioterapia se consolida como una profesión liberal del área de la salud; esta ley replanteó adicionalmente las responsabilidades y competencias del fisioterapeuta y actualizó los ámbitos de acción que le competen a la luz de las concepciones y los requerimientos contemporáneos en salud y seguridad social; así, el objeto de trabajo del fisioterapeuta fue claramente definido por la nueva reglamentación mediante el establecimiento de las funciones propias del ejercicio profesional (Herrera, Rivera, Prada \& Sánchez, 2004).
Es necesario considerar al fisioterapeuta como profesional del área de la salud que requiere tener unas condiciones relacionadas con su desempeño laboral, su bienestar y su calidad de vida adecuadas para desempeñarse en favor de la población en todos los ámbitos, campos y áreas de actuación que la ley le confiere. Actualmente el Fisioterapeuta es un profesional competente para desempeñarse con suficiencia en todas las áreas definidas en el perfil profesional, aceptadas universalmente por la comunidad profesional (Herrera, et al., 2004).

El Programa de Fisioterapia de la Universidad CES, luego de once años de desarrollar un enfoque de formación en Fisioterapia que responda a los estándares de calidad nacional e internacional y a las necesidades y características del contexto regional de la salud (Arenas \& Múnera, 2013), continúa con la formación de profesionales universitarios bajo los marcos legislativos que orientan la salud y la Fisioterapia en Colombia (SENA, 2008).

Hasta la fecha se sabe que la profesión de fisioterapia está en crecimiento y el Programa de Fisioterapia de la Universidad CES, según el reporte sobre sus graduados, para el año 2014 contaba con 167 egresados quienes según los empleadores, tienen un desempeño profesional altamente valorado (Arenas \& Múnera, 2013).

Se sabe que la profesión de fisioterapia está en crecimiento, pero no se tiene claro en qué condiciones están los egresados, cuáles creen ellos que son las condiciones que limitan o facilitan el desempeño de su profesión; la Universidad CES no cuenta con una investigación que muestre lo anterior y por tal motivo no se pueden desarrollar estrategias para fortalecer las condiciones que favorecen el ejercicio profesional o menguar las que están restringiendo el desarrollo de este. Por todo lo anterior se hace necesario una investigación que responda cuáles son aquellas condiciones que, desde la perspectiva de los egresados de dicha universidad, favorecen el ejercicio profesional de la fisioterapia. 


\section{MATERIALES Y MÉTODOS}

\section{Enfoque y tipo de investigación}

Se realizó un estudio cualitativo de alcance exploratorio cuyo propósito fue explorar las condiciones que favorecen el ejercicio profesional de los fisioterapeutas egresados de la Universidad CES, Medellín (Colombia). En el estudio participaron 4 egresados, seleccionados a partir de las siguientes características: Egresados (as) del Programa de Fisioterapia de la Universidad CES, que se desempeñaran en los diferentes ámbitos de actuación del fisioterapeuta y que participaran voluntariamente de la investigación.

\section{Participantes}

Para la convocatoria se tomaron las bases de datos de los egresados del programa, y a través de correo electrónico se envió la invitación a todos los registrados; asistieron 4 egresados. Participaron dos hombres y dos mujeres, todos con menos de 5 años de experiencia laboral; el $50 \%$ ya finalizó su formación de posgrado y el otro $50 \%$ la tiene en curso; un participante se desempeña como docente, dos, en el campo terapéutico y de rehabilitación, y uno se encuentra desempleado.

\section{Proceso de generación y análisis de la información}

La generación de datos fue por medio de una entrevista semiestructurada y un grupo focal; a través de ellos se indagó acerca de las condiciones laborales, específicamente sobre el acceso al sistema laboral, el salario y el tipo de vinculación; las condiciones de ejercicio profesional, que abarcaron la adecuación del ejercicio al perfil, la satisfacción percibida con el ejercicio profesional y el reconocimiento en los equipos de trabajo del saber y el criterio del fisioterapeuta.

Asimismo, se indagó sobre el bienestar percibido en el trabajo, la participación política y gremial, y las expectativas frente a la vida profesional. Cabe aclarar que la presente investigación hace parte del estudio nacional sobre las condiciones de trabajo y salud de los fisioterapeutas en Colombia, específicamente en el proceso de diagnóstico institucional que, para el caso de la Universidad CES, se realizó tanto de manera cuantitativa como cualitativa; las categorías iniciales fueron dadas como lineamiento nacional, y a partir de ellas se construyeron unas emergentes propias para los participantes de la Universidad CES.

Los contenidos generados fueron analizados con apoyo del Atlas TI 5.2 y con las orientaciones metodológicas que la teoría fundada ofrece en términos de codificación de la información, pasando así por codificación abierta y selectiva, lo que permitió fragmentar la información y construir categorías que permitieron su ordenamiento (Strauss \& Corbin, 2002). El estudio fue aprobado por el comité de ética de la Universidad CES y todos los participantes firmaron el consentimiento informado, a través del cual se aseguró la protección de la intimidad, el anonimato, la libre participación y la voluntariedad de los entrevistados.

\section{RESULTADOS}

Los resultados de la investigación se ordenan en torno a unas categorías emergentes que hablan de las condiciones que favorecen el ejercicio profesional del fisioterapeuta según la perspectiva de los egresados del Programa de Fisioterapia de la Universidad CES. La principal categoría emergente fue la de amor a la fisioterapia, que no había sido contemplada por el estudio nacional y que para el caso de la Universidad CES resultó ser fundamental por poner de presente unas condiciones propias del profesional, endógenas, que no dependen ni del contexto ni de la universidad de donde sea egresado, sino de una posición personal frente a la profesión que se eligió.

\section{Actualización profesional permanente i̇una necesidad?}

La cualificación profesional permanente se refiere a la actualización de contenidos y técnicas propias del desarrollo de la profesión una vez terminado el pregrado; esta cualificación puede hacerse a través de dos vías: una, referida a la formación desde las actividades de educación continua, ofrecidas por las instituciones de educación superior; otra, a la realización 
de estudios de posgrado. La educación continua se constituye para los participantes en un complemento para la vida laboral, un espacio donde pueden apropiar herramientas teóricas y prácticas requeridas en los escenarios en que desarrollan su profesión; además, esta formación les posibilita un acercamiento a las demás profesiones con que hacen equipo de trabajo, y ello favorece el posicionamiento de la fisioterapia a través de la labor de los fisioterapeutas:

"los mismos fisioterapeutas que quieren modificar la visión que hemos tenido hasta hace ciertos años, entonces es el fisioterapeuta que también quiere estudiar, que está en la búsqueda, que se esfuerza por continuar ese proceso educativo, yo creo que eso es fundamental y que ahora lo hemos hecho muy bien"

Con respecto a la realización de estudios de posgrado, para los participantes éste es un requerimiento aún incipiente, pues la demanda del contexto ha estado básicamente orientada a la fisioterapia general, aunque se reconoce que a nivel gremial no se ha dado a este nivel de formación el carácter de necesario:

“...tradicionalmente hemos sido una profesión que muchas instituciones requieran fisioterapeutas generales, por decir algo, y no hemos dado la necesidad de la especialidad o de la educación pos-gradual..."

Los niveles de especialización y maestría se consideran requeridos por ciertos campos de actuación, tales como la docencia, la investigación y cuidado crítico:

“...no hemos dado la necesidad de la especialidad o de la educación pos-gradual, a excepción de estancias por ejemplo como la de los cuidados intensivos que de pronto ahi sí se requiere un poco más de este tipo de educación..."

Tanto la educación continua como la realización de posgrados, constituye para los participantes una forma de enfocar el ejercicio profesional en un área específica, esto se traduce en mayor especialización y posicionamiento de la profesión en ámbitos laborales particulares, favoreciéndose de tal modo el ejercicio de la fisioterapia y la consolidación de la misma en el medio como una opción de intervención claramente diferenciada.

\section{Construcción del rol profesional: más que una asignación}

La construcción del rol profesional, la definición de funciones y el establecimiento de lo esperado en los egresados de fisioterapia, es para los participantes una condición que favorece su ejercicio; el rol está mediado por el posicionamiento en el medio de la universidad de donde es egresado y por el nivel de autonomía que tengan los profesionales en su ejercicio.

Con respecto a la universidad, señalan que es ella quien da las bases de la formación tanto a nivel teórico como práctico y actitudinal:

"Es muy importante la educación que uno recibe pues digamos que esa es la mentalidad que uno va cogiendo de acuerdo a lo que vea y uno que ve lo que hace los profesores, lo que hace, lo que uno hace en las prácticas"

"Hay un chip que juega un papel importante, un papel importante porque aparte del pensum, de la parte académica en sí, que tenga ese pensamiento me parece supremamente importante".

Esa "mentalidad" transmitida por la institución, que habla de una marca de los profesionales que los distinguen como parte de ella, en el caso de la Universidad CES se sintetiza en "excelencia”, tal como lo afirman los participantes:

"Yo primero no lo miro desde la facultad sino desde la universidad; el hecho de que vos te metan el compromiso con la excelencia y que estés cinco años o muchos más en una institución y te estén metiendo eso, eso te programa de una forma que a nivel laboral en muchos va a generar un impacto positivo"

A lo anterior se suma la orientación del currículo en el que son formados:

El punto de vista de currículo es un impacto muy positivo porque digamos que eso es lo que está haciendo 
el cambio en la profesión, o sea, universidades y facultades como esta es las que generan ese tipo de cambio.

Frente a lo cual los participantes resaltan la diversidad de asignaturas, áreas y campos de actuación que se abordan durante el pregrado, lo que les permite egresar con competencias básicas para el desempeño en múltiples escenarios:

"Es brindarle las herramientas al estudiante desde la parte terapéutica, intervención, de evaluación, de ciencias básicas, pero también tienen la otra parte la dimensión de la salud, las materias que tienen durante su formación desde la salud pública hasta la conformación de una política pública, entonces tener esas herramientas también yo creo que impactan en eso, que a no todos les guste, no, es que lo chévere y lo rico de esto es que a cada uno le gusta algo específico y que sea muy bueno alli”.

El acercamiento a la práctica profesional desde el pregrado es también un aspecto resaltado por los participantes:

"Nosotros somos una profesión con un componente práctico muy importante y que digamos que eso se potencializó en esta carrera, de pronto otras facultades no hacen tanto énfasis, entonces nosotros a la hora de graduarnos es como si hubiéramos trabajado mucho tiempo, o sea, no tuvimos que hacer un cambio de mentalidad, sino que ya seguimos trabajando".

Finalmente, el rol del docente en la formación de fisioterapeutas es un aspecto que favorece el posicionamiento de una universidad en el medio:

"Todo ese esfuerzo que uno hizo en las prácticas con los profesores, en todo lo que ellos nos decían, era cierto... todos esos días me tocaba estar sola, me tocaba dos y tres dias sola sin apoyo profesional que era la que estaba supuestamente pendiente de mí, yo vi que uno se lo lleva, todo eso sirve y todo ese esfuerzo, toda esa insistencia de los profesores estudie esto, pilas con esto, cuidado con esto, uno lo aplica y entonces uno ahi se da cuenta de que sí funciona y que realmente todo ese esfuerzo que hacen los profesores no es en vano".

Así, la filosofía institucional, el currículo del pregrado y la práctica docente, son los elementos coayudanres a que una institución de educación superior se posicione en el medio como formadora de fisioterapeutas de calidad; ello, sumado a la autonomía que los profesionales tienen para el ejercicio en diferentes áreas, contribuye a la construcción del rol profesional. $\mathrm{Al}$ respecto, los participantes consideran fundamental la posibilidad de tomar decisiones de tratamiento de acuerdo con argumentos profesionales sólidos y el trabajo en equipo, con otros profesionales, en el que esas decisiones puedan comunicarse, enriquecerse y consolidarse a favor de una mejor intervención.

Cada profesión tiene definido un rol, es decir, delimitadas las funciones y actitudes que le corresponden (Universidad del país Vasco, s. f.); aunque éste rol no es estático, si es bastante estable en tanto está regulado. Los profesionales, de acuerdo con los participantes, tienen una responsabilidad fundamental en el mantenimiento y enriquecimiento de su rol:

“...yo creo que, si desde la universidad no concientizan a los estudiantes de dar una buena importancia a la carrera, de dar una buena imagen, de no sé, en el área laboral de dedicarse realmente a lo que es la carrera, la rehabilitación física como tal en el caso musculoesquelético, todo se va a ir perdiendo y la fisioterapia cada vez va perdiendo como la importancia que hasta ahorita ha ganado..."

“...es la expectativa de "voy a ser el mejor y yo quiero ser el mejor y voy a representar muy bien lo que yo soy en mi profesión...".

"La lucha que todos lo que quieran aportarle a la profesión deben hacer, eso no es una lucha de poquitos sino de muchos, pero de muchos que quieren ser buenos y diferentes porque eso también ha pasado, la gente se dedica a criticar y no nos autoevaluamos”.

Es también importante para ellos el hecho de que, aunque el rol está previamente definido para la 
profesión, cada profesional construye su lugar gracias a una condición, que fundamentalmente, es proporcionada por las instituciones donde se trabaja: la autonomía. Esta se refiere a la posibilidad de tomar decisiones sustentadas en criterios teóricos y prácticos propios de la profesión, esto a su vez se traduce en un claro posicionamiento frente a otros y en el fortalecimiento del trabajo interdisciplinario:

“...yo desde el punto de vista clínico, cierto, pues en la relación con el paciente, en su tratamiento, en su manejo soy muy autónomo...”.

En este marco, se destaca para el foco de interés las relaciones entre los profesionales de la Fisioterapia y otros profesionales del sector, identificando el impacto que dichas relaciones tienen para la práctica y el bienestar profesional (Asociación Colombiana de Fisioterapia, ASCOFI; Asociación Colombiana de Facultades de Fisioterapia, ASCOFAFI; Colegio Colombiano de Fisioterapeutas, COLFI; Asociación Colombiana de Estudiantes de Fisioterapia, ACEFIT, 2015).

"él lo puede hacer así, a mí no me parece yo también conozco de este o he aprendido este y siento que a mí me funciona más, pero eso no quiere decir que yo me enfrente con el otro o que la relación este tensa (...) Son diferentes maneras de abordarlo y que también dentro del aprendizaje está el respeto por lo que él hace y aprender también de él, que creo eso es un verdadero trabajo en equipo".

"En una parte hay ciertas personas que manejan un tema más que por ejemplo que lo que manejo yo entonces que ATM, que piso pélvico, que deporte, que ortopedia, entonces como cada quien tiene su área específica, entonces de todos nos retroalimentamos y es algo muy positivo".

A la construcción del rol, está también asociado el reconocimiento social que tengan la universidad de donde se egresa, y el tipo de organización donde se labore, pues de ello depende que las condiciones de trabajo sean óptimas y que permitan no sólo la calidad de las prácticas de los profesionales, sino también su desarrollo personal.

\section{Condiciones de Trabajo}

Se refiere al ordenamiento propiamente dicho de la práctica profesional que la regula, donde se hace necesario caracterizar al sujeto de la actuación; los ambientes, equipos e instrumentos de trabajo; las actividades a realizar y su organización en términos de jornadas, salarios, formas contractuales, prestaciones, entre otras (ASCOFI, et. al, 2015).

“...lo que yo considero algo ideal para trabajar, o sea, las instalaciones son las adecuadas, hay protección hacia el empleado en este caso en salud, hay fomento a que la persona que esté trabajando crezca profesionalmente, cierto, no solamente lo que está haciendo o bueno vaya atienda tantos pacientes, sino siga creciendo en lo suyo, sígase especializando, siga haciendo este tipo de educación posgradual por ejemplo, se da la oportunidad de o por lo menos en el espacio en que laboro, trabajar o atender pacientes digamos que con las condiciones con las que yo me siento cómodo trabajando...".

En general, se percibe un panorama de dificultades para la inserción de los fisioterapeutas en el mundo laboral bajo condiciones que permitan el desempeño profesional de calidad; por un lado, la cuestión de los salarios es álgida, y por el otro, la exigencia de experiencia, que es por demás difícil de acumular puesto que las ofertas laborales son escasas, se considera también un obstáculo para el desarrollo laboral:

"El mercado está malo, hay alteraciones o malos pagos en algunos profesionales" [...] "La principal la contratación por cooperativa y el enmascaramiento financiero que hay detrás de eso para que el hospital no pague nada al empleado" [...] “... los trabajos que ofrecen para los egresados los jefes se aprovechan de esa condición y son con sueldos demasiado bajos..." [...] "...vos salís como general, empiezas a trabajar en un área específica, en una institución específica y te especializas, haces la inversión, dedicas el tiempo y asi seas especialista te siguen pagando exactamente lo mismo..." [...] “... yo no tengo experiencia y necesitan que tengan por ahi dos años de experiencia, tres años de experiencia, y por eso no lo aceptan a uno...”. 
La cantidad de pacientes atendidos por hora, en el caso de los profesionales que trabajan en el área de la salud, es otro factor que tiene alta relevancia a la hora de valorar las condiciones de trabajo:

"mientras en un hospital público uno ve 10 o 12 pacientes por hora, en una parte privada de pronto a que se llegue máximo a 4 o 5 por hora...”.

Sobre las condiciones de trabajo los profesionales no tienen injerencia directa, aunque nuevamente señalan que pueden tener parte de responsabilidad en lo que laboralmente les sucede:

Otra de las cosas que obstaculiza mucho es que el fisioterapeuta desconoce los derechos y deberes que tiene como trabajador, entonces ese desconocimiento los lleva a que pasen por encima de mí y yo no sé si eso es legal o no es legal, si esta propuesta de trabajo que me hicieron pues es conveniente o no, entonces yo creo que el desconocimiento también tiene un alto obstáculo para la profesión.

Así, las condiciones de trabajo para los fisioterapeutas participantes de esta investigación, se componen de elementos del contexto que se perciben en este caso como dificultades para el ejercicio, pero que son susceptibles de mejora si se generan procesos de empoderamiento del gremio. Igualmente, dichas condiciones del egresado, formación profesional y experiencia que a su vez requieren de la práctica profesional para mejorar las competencias del perfil de egreso hasta lograr un perfil profesional que le permitan desempeñar su profesión en condiciones de trabajo adecuadas.

\section{Amor por la fisioterapia: de estudiar fisioterapia, a ser fisioterapeuta}

Una de las condiciones que para los participantes favorece el ejercicio profesional es el amor por la fisioterapia; amar lo que se hace, sentir gusto y sentido de pertenencia por la profesión, se consideran fundamentales para el buen ejercicio:
Yo creo que es de encontrarle el gusto, el gusto a lo que uno quiere hacer, uno le encuentra ese gusto pues de pronto se puede ir yendo con más facilidad, porque definitivamente a uno le tiene que gustar lo que hace (P1).

Los participantes manifiestan amor por la profesión y disfrute por lo que hacen, haciendo de la fisioterapia un asunto de importancia para la vida:

Yo amo ser fisioterapeuta, a mí me gusta y lo disfruto (P1) [...] Fisioterapia yo no la cambio por nada del mundo (P2).

El amor por lo que se hace es entonces una condición intrínseca que se traduce en un motor para la formación continua, la especialización, el desempeño profesional de calidad y la lucha por posicionar a la fisioterapia como una profesión autónoma, independiente y con sustento científico. Las dificultades que se imponen desde el mercado no restan fuerza a ese sentimiento que para los participantes no es sólo de identificación con una profesión, sino de construcción de una identidad personal.

\section{DISCUSIÓN}

Para identificar las condiciones laborales de los fisioterapeutas egresados de la Universidad CES se hace necesario caracterizar al sujeto de la actuación, los ambientes, equipos e instrumentos de trabajo, además de las actividades a realizar y su organización (ASCOFI et al, 2015); todas estas permiten reconocer las condiciones que inciden en su ejercicio profesional, las cuales hacen referencia a asuntos que favorecen $u$ obstaculizan su desempeño profesional, donde algunas condiciones se presentan asociadas al contexto y otras pueden nombrarse como de origen endógeno.

Para el egresado de Fisioterapia de la Universidad CES las condiciones vinculadas con el contexto, no son consideradas de incidencia personal y están relacionadas con el colectivo profesional, del cual se espera su asociación fortalecida en beneficio del ejercicio profesional que se requiere para la Fisioterapia en Colombia. 
Asociado a asuntos legislativos y de organización estatal para el empleo, los egresados del programa por su propia acción no pueden incidir sobre las condiciones laborales de su ejercicio profesional y para ello es necesario que como profesionales se afilien a las Asociaciones en Fisioterapia del país, quienes tienen la tarea de velar por un ejercicio profesional digno, a la vez que apoyan el fortalecimiento y participación activa para el mejoramiento de las condiciones laborales, tarea que ha dado sus frutos y hoy presenta un plan de trabajo conjunto para la Fisioterapia en Colombia (ASCOFI, et. al, 2015), el cual requiere que las organizaciones sigan creciendo y logren los objetivos que individualmente no se tendrán.

La formación de pregrado que reciben los egresados en fisioterapia está relacionada con los procesos formativos durante la vida universitaria y allí están asociados elementos reconocidos por el Ministerio de Educación Nacional, como el prestigio de la Universidad de donde egresa, la calidad del programa de pregrado y la formación integral sumado a los valores diferenciadores que fortalecen el perfil de egreso en cada programa; estos asuntos son reconocidos por las entidades que los emplean.

Lo anterior no ocurre con la formación de posgrado, la cual solo en algunas áreas recibe una remuneración mayor asociado al postgrado; esto sumado a la crisis que vive nuestro sistema de salud desestimula la formación posgradual de los fisioterapeutas, la cual profundiza en áreas y campos profesionales necesarios para la atención a poblaciones específicas. Es menester que sea reconocida la labor del fisioterapeuta con formación de postgrado, el cual es necesario en áreas de alta complejidad con competencias específicas en áreas donde la formación del pregrado no profundiza.

Como parte de los procesos de mejoramiento y para la renovación del registro calificado, el plan de estudios del pregrado de fisioterapia se fortaleció en el área curricular administrativa donde se espera que los egresados adquieran competencias para integrar equipos de trabajo interdisciplinarios, utilizando herramientas de administración y gestión para la transformación de su entorno profesional, laboral y social, lo cual les permite tener los conocimientos, desempeñarse y ser fisioterapeutas que puedan incidir en su entorno profesional; asunto que parece utópico pero ¿qué sería de la excelencia sin las utopías?

De manera endógena aparecen condiciones en los egresados de Fisioterapia de la Universidad CES que están asociadas a su decisión personal de formación, como lo plantea la Confederación Mundial de Fisioterapia (WCPT 2015) en sus principios profesionales, al desarrollo profesional requerido para estar a la vanguardia en temas de actualidad profesional, los cuales se ven representados en las mejores decisiones en favor de los pacientes y comunidades donde interviene.

La educación de pregrado debe tener un vínculo permanente con la educación continua, ya que lo establece la Ley 528: la capacitación y la actualización permanente de los fisioterapeutas identifican individualmente o en su conjunto el avance del desarrollo profesional. Por lo tanto, la actualización constituye un deber y una responsabilidad ética (Ley 528 de 1999), la evidencia a nivel mundial para tomar mejores decisiones en favor de la salud de los pacientes requiere conocimientos vanguardistas.

La autonomía e independencia del fisioterapeuta, como se expresa en la Ley 528 de 1999 , son la base fundamental del ejercicio profesional y para ello es necesario que el criterio clínico, la toma de decisiones y el trabajo en equipos interdisciplinarios sea una fortaleza en la formación de los profesionales, y de esta forma su ejercicio profesional sea adecuado según los parámetros que nacional e internacionalmente se esperan de la profesión; ese ejercicio profesional en equipos de trabajo debe ser leal y respetuoso para que las relaciones sean vínculos profesionales que permitan el ejercicio profesional autónomo (Ley 528 de 1999).

Al considerarse la Fisioterapia como una profesión liberal es necesario comprender que estas profesiones se caracterizan según Weber, por exigir competencias conceptuales, procedimentales y actitudinales. Así, las profesiones liberales se aprenden en la práctica y en un sistema formal de educación ya que implican la formación teórico-conceptual; también implican 
que la práctica sea socialmente responsable, donde es tan importante el desarrollo y apropiación del conocimiento como el servicio social que se presta.

En estas condiciones propias de los egresados, emerge el amor por la fisioterapia; éste, introduce un elemento sorpresa en la investigación pues habla de asuntos que van más allá de lo gremial, lo institucional o la capacitación; concernientes a una relación íntima del fisioterapeuta con su profesión, la cual le permite una identidad personal, pues la fisioterapia no se toma como una profesión que se estudia, sino como algo que define el ser.

Esto, para los participantes, puede ser el motivo por el cual, pese a las dificultades del entorno en términos de lo laboral, sigan comprometidos con la profesión y con su desarrollo como profesionales; porque la fisioterapia no es un accesorio legitimado en un título sino un elemento constituyente del ser.

Esta cuestión hace reflexionar en torno a la denominada "formación integral", que en la Universidad CES es un eje fundamental ¿es posible educar el ser? ¿De qué manera se haría? ¿Cuáles son las condiciones que hacen posible el amor por la fisioterapia? ¿Esta relación de amor con el hacer y el saber del fisioterapeuta pueden promoverse? Estas cuestiones atañen entonces a aspectos curriculares sobre los que vale la pena seguir indagando.

\section{CONGLUSIONES}

A través de las entrevistas y el grupo focal realizado, fue posible explorar las condiciones que para algunos egresados de fisioterapia de la Universidad CES, favorecen el ejercicio profesional. Fundamentalmente son condiciones del mercado laboral, el equipo de trabajo y la formación recibida durante el pregrado; estas condiciones, sin embargo, no aseguran por sí solas un ejercicio profesional satisfactorio: el amor por la fisioterapia, sentirse fisioterapeuta y vincular el ser con el ejercicio, emergió como una condición personal que, relacionada con las del medio, favorecen la actuación profesional.
Dado el alcance exploratorio del estudio, vale la pena aclarar que los resultados presentados no pueden ser generalizados; es necesario continuar profundizando a través de otras variables tales como años de experiencia, ámbito de desempeño, instituciones en las que se labora, y en general, experiencias más específicas en el ejercicio profesional. La categoría de amor por la fisioterapia puede también continuarse trabajando.

Queda también abierta la opción de llevar a cabo investigaciones que se realicen con un mayor número de participantes, con más años de experiencia (incluidos los mismos que participaron esta vez) y con otras miradas de sus condiciones laborales que representen diferentes campos, áreas, contextos y ámbitos de la Fisioterapia.

\section{REFERENCIAS}

Arenas, C., \& Múnera, C. (2013, septiembre). Universidad CES, Indicadores Extensión. Universidad CES.

Asociación Colombiana de Fisioterapia, ASCOFI; Asociación Colombiana de Facultades de Fisioterapia, ASCOFAFI; Colegio Colombiano de Fisioterapeutas, COLFI; Asociación Colombiana de Estudiantes de Fisioterapia, ACEFIT (2015). Perfil profesional y competencias del fisioterapeuta en Colombia. Recuperado de: https://www.minsalud.gov. co/sites/rid/Lists/BibliotecaDigital/RIDE/VS/TH/Perfilprofesional-competencias-Fisioterapeuta-Colombia.pdf

Cerpa, J (2010). Análisis de las acciones del fisioterapeuta en la ejecución de la política de salud pública en el hospital de Suba, empresa social del Estado II Nivel. Recuperado de: http://cdim.esap.edu.co/BancoMedios/Documentos\%20 $\mathrm{PDF} /$ proyecto $\% 20 \mathrm{de} \% 20$ investigación $\% 201 \mathrm{a} \% 20$ fisioterapia $\% 20 \mathrm{en} \% 20$ salud $\% 20$ publica.pdf

Congreso de Colombia. Ley 528 de 1999. Recuperado de: http://www.mineducacion.gov.co/1621/articles 105013 archivo_pdf.pdf

Chillón, R., Rebollo, J., \& Meroño, J. (2008). El pensamiento histórico-filosófico y los fundamentos científicos en el estudio de la fisioterapia. Revista Fisioterapia, 7(2), 5-16. Recuperado de: http://www.ucam.edu/sites/default/files/revista-fisio/01el_pensamiento_historico-filosofico_y_los.pdf

Gallego, T. (2007). Bases teóricas y fundamentos de la fisioterapia. Madrid: Médica Panamericana.

Herrera, E., Rivera, L, Prada, A., \& Sánchez, D. (2004). Evolución histórica de la fisioterapia en Colombia y en la Universidad Industrial de Santander. Salud UIS, 36(1), 21-31. 
Ministerio de Educación Nacional de Colombia. (2016). Sistema Nacional de Información de la Educación Superior SNIES. Recuperado de: http://www.mineducacion.gov. co/1621/w3-article-156293.html

Servicio Nacional de Aprendizaje, SENA. (2008). Caracterización de la profesión de fisioterapia en Colombia. Recuperado de: http://repositorio.sena.edu.co/bitstream/11404/2139/1/3063.pdf
Strauss, A., \& Corbin, J. (2002). Bases de la investigación cualitativa. Técnicas y procedimientos para desarrollar la Teoría Fundamentada. Medellín, Colombia: Editorial Universidad de Antioquia.

Universidad del país Vasco. (s. f.). Teoría del rol. Rol profesional. Recuperado de: http://www.ehu.eus/xabier. zupiria/liburuak/relacion/2.pdf 